\title{
A new adaptive test for paired data for small to moderate sample sizes.
}

\begin{abstract}
When carrying out data analysis, a practitioner has to decide on a suitable test for hypothesis testing, and as such, would look for a test that has a high relative power. Tests for paired data tests are usually conducted using t-test, Wilcoxon signed-rank test or the sign test. Some adaptive tests have also been suggested in the literature by O'Gorman, who found that no single member of that family performed well for all sample sizes and different tail weights, and hence, he recommended that choice of a member of that family be made depending on both the sample size and the tail weight. In this paper, we propose a new adaptive test. Simulation studies for $n=25$ and $n=50$ show that it works well for nearly all tail weights ranging from the light-tailed beta and uniform distributions to $t(4)$ distributions. More precisely, our test has both robustness of level (in keeping the empirical levels close to the nominal level) and efficiency of power. The results of our study contribute to the area of statistical inference.
\end{abstract}

Keyword: Modified Wilcoxon test; Adaptive test; Linear rank test. 